\title{
CIENCIA, LITERATURA, ARTE Y RELIGIÓN EN IDOMEYKO
}

\section{MANUEl. JOFRÉ IBERRIOS*}

El 8 de enero cle 1866, a la edad de 62 años, Ignacio Domeyko pronuncia su Discurso de Incorporación a la Facultad de Filosofía y Humanidades de la Universiclad de Chile. En la ocasión, pasa a ocupar el sitial que anteriormente le había correspondido al Primer Rector de la Universidad de Chile, Andrés Bello, fallecido en 1865.

Domeyko es miembro de la Universidad de Chile desde 1843 y del Consejo Universitario desde 1846. Llegará a ser posteriormente elegido como el tercer Rector de la Universidad de Chile, luego de Manuel Antonio Tocornal, y estará en dicha función desde el 29 de septiembre de 1867 hasta el 30 de mayo de 1883.

Antes de entrar al análisis del Discurso de Incorporación, es indispensable aclarar que en el hombre Domeyko coexisten dos sujetos. Primero, un hombre de acción, un realizador; y segundo, un intelectual, un sabio. El estudio a continuación se concentra en el hombre de letras. Este investigador y académico se manifiesta fundamentalmente en su escritura.

En consecuencia, se debe diferenciar entre el hombre Domeyko y su producción intelectual, conservada en uno de los principales productos de su gestión: su escritura. Interesa examinar pues no la persona sino que los dispositivos, las matrices, las relaciones y articulaciones de su pulsión escritural. En este sentido, esta monografía se centra más en su obra que en su vida.

\section{EL IDISCURSO IDE INCORPORACIÓN}

El texto de Ignacio Domeyko se titula "Ciencias, literatura y bellas-artes, relación que entre existe". Discurso de Ignacio Domeyko en su

\footnotetext{
- Manuel Jofré Berríos, Universidad de Chile.
} 
incorporacion a la Facultad de Filosofía y Humanidades, leída en la sesión del 8 de enero de 1866. Se encuentra impreso en Santiago de Chile, Imprenta Nacional, Calle de La Moneda $N^{\circ} 46$, y está fechacla la edición en enero de 1867.

Con este I)iscurso, Ignacio Domeyko se convierte en Miembro de número de la Facultad de Filosofía y Humanidades, tal como lo establece el decreto presidencial del 11 de junio de 1866, "en reemplazo del finado Miembro don Andrés Bello..., quien ha sido elegido por la expresacla Facultad", en nota firmada por Federico Errázuriz, publicada en los Anales de la Universidad de Chile, tomo XXVIII, de 1866, página 433.

Esta intervención de I omeyko, de 23 páginas de extensión, será aquí comentada utilizando una aproximación textual consistente en un conjunto de nociones provenientes de la teoría y análisis de discurso. Esto se realizará en el marco de los estudios culturales para mejor abordar las matrices de senticlo epocales y las sensibiliclades histórico-filosóficas plasmadas en su discurso.

En síntesis, pueden distinguirse cuatro momentos en el Discurso de incorporación: primero, una introducción, centrada en la comparación entre Bello y Domeyko que realiza éste último; segundo, una descripción positiva del valor de la ciencia; tercero, lo positivo de la imaginación poética; y cuarto, el valor cle la religión como climensión totalizadora.

\section{EL CONTRASTE COMPARA'TIVO ENTRE BSELLO Y DOMEYKO}

El Discurso de Incorporación está dividido en diez secciones, de diferente extensión, no numeradas. En la primera sección, de carácter introductoria, descle el inicio, Domeyko se define como dedicado a lo largo de su vicla a la enseñanza pública. Inmediatamente, a continuación, establecerá un paralelo constante entre Andrés Bello y él mismo.

Ignacio I omeyko destaca el don de habla de Andrés Bello, tan contrapuesto al suyo, que califica como cluro. Domeyko, a esa altura de su vida maneja por lo menos ocho lenguas: el polaco, el lituano, el alemán, el ruso, el latín, el francés, el inglés y el espanol. Mientras Bello era escritor y filósofo, y conocedor del corazón humano, él, 1)omeyko sólo conoce las peñas y el globo material sobre el cual toclos nos hemos asentado. Domeyko se define a sí mismo geográficamente como 
alguien que nació en la rivera del Niemen, bajo cielos tan duros como su lengua. Este es un determinismo geográfico.

Bello luchó por la libertad contra la colonización, y junto con Bolívar, por la Independencia. Para Domeyko, la Independencia significaba que la "instrucción (fuera) accesible a todas las clases" y que se manifestarán "las letras y las ciencias en progreso". En IBello, según Domeyko, se unía el métoxlo científico más la poesía del estilo (en una suerte de integración del contenido a la forma).

El retrato que hace de sí mismo Domeyko no deja de ser fuerte. Él, como Bello, luchó por la libertad de su pueblo contra los invasores rusos, con un éxito fugaz. Su pueblo debió realizar por honra una seguncla guerra que sabían que no ganarían. Su tierra está nuevamente dominada. Así establece Domeyko esta comparación entre los bordes de clos continentes, América del Sur y centro de Eurasia.

\section{BELLO COMO INTELECTUAL I TEGRAL}

Domeyko proporciona un retrato de Bello de primera mano. Ambos mantuvieron muchas conversaciones, deteniéndose especialmente sobre las ciencias naturales o ciencias de la observación. Bello, aunque humanista, era también un científico, porque conocía la ciencia moderna.

En especial, Domeyko destaca que Bello no tuvo una predilección por una sola disciplina. Bello era un genio "más universal", atento al "conjunto de las ideas y los conocimientos", conocedor de varias disciplinas, con una especialidad unida a una "tendencia general de la humaniclad".

En algunas conversaciones de Bello con Domeyko, el primero se refería a la relación entre bellas artes, literatura, ciencia y moral, y recordando todo ello, Domeyko declara que el tema de su Discurso Inaugural será la relación entre la ciencia y la literatura. Con ello, está claramente en el terreno de las humanidades, no en un tópico científico.

\section{EL GRAN MOIDELO ENCICLOPÉI)ICO: HUMBOLDT}

Según I)omeyko, el primero que enlazó la ciencia con la literatura fue Humboldt, en los dos tomos de su obra Cosmos, al hacer confluir "la observación, el cálculo y la vía experimental” con lo poético de la literatura, la inteligencia y el sentimiento. Se trata del mundo exterior, abar- 
cado por los sentidos y el mundo interior, donde reside el sentimiento, la imaginación y la inspiración poética.

Domeyko propone, citando a Humboldt, como una posición epistemológica, una teoría del reflejo, según la cual en el munclo interior se refleja el mundo exterior. Claramente, esta teoría presenta como causa primera el mundo externo.

Según Domeyko, en su obra Humboldt buscó "la creación entera en su conjunto". Esta ansiedad hegeliana de la totalidad es lo que de él destaca, como propio de la imaginación poética. Justamente, en su obra Cosmos, se propuso la unión del sentimiento más la inteligencia, la razón más la imaginación y el cálculo más la inspiración. Podría decirse aquí que los icleales neoclásicos se integran a los ideales románticos, en esta formulación.

\section{LA FUNCIÓN DE LA CIENCIA MOIDERNA}

Humboldt, Bello y Domeyko han estado pues atentos a la suma de lo bello más lo verdaclero en la naturaleza y en las obras y tendencias de los hombres. Sobre todo, y aquí comienza a desarrollar Domeyko su propia argumentación, para él, los enormes descubrimientos de la ciencia abren nuevos campos a la imaginación: "la ciencia moderna abre al hombre de letras, al poeta, al artista, espacios invisibles al vulgo".

1)omeyko, como Bello, sigue a Humboldt, en todo este razonamiento. La contemplación intelectual poética ha ganado en cuanto a los objetos que incorpora el munclo. Las ciencias naturales y experimentales se han clesarrollado en el siglo XVIII y XIX, al mismo tiempo que se manifestaba una fascinación por la naturaleza, expresada en el arte y la literatura.

La unión de la ciencia y la literatura se manifestará tardíamente. Domeyko dice que hay que reconocer primero que los hombres de letras poseen conocimientos científicos superficiales y que los naturalistas rara vez se elevan al "conjunto de los fenómenos del universo". Lo científico y lo artístico escasamente se dan juntos en un creador, pero a esta unión aspira "el genio del hombre".

"El hombre de letras, el poeta, el artista, (son) guiados, impulsados por el hombre de ciencia moderna". Lo que se busca como utopía es que ambas lógicas coexistan en "una sola inteligencia". Podría decirse que se presenta una idea neoclásica de la armonía, integradora del sentimiento y la razón, tema de la filosofía germana. 
En el Discurso, Domeyko da prueba de su sensibilidad al construir cuadros pictórico-verbales acerca de algunas regiones. Pone especial énfasis en describir la naturaleza del trópico, de manera similar a como lo hizo Andrés Bello en su "Oda a la agricultura de la zona tórricla". Implícitamente, está estableciendo con él una nueva comparación contrastiva.

Para Domeyko, Bello es a la vez un naturalista, un hombre de imaginación y un pensador profundo. Por eso destaca, en su obra, descle su punto de vista, bellezas geográficas que sólo una ciencia puede revelar.

También hace presente Domeyko una visión del polo norte, que según él descubrieron los islandeses cinco siglos antes que Colón. Así, ha pasado de lo tropical a lo glacial, pues para él, la naturaleza tiene "en su forma la belleza del universo". La concepción filosófica de Domeyko es compleja, basándose en criterios formalistas, paísajísticos y visuales en lo estético. Bello, venezolano, describió el trópico; Domeyko, polaco, describe lo glacial de su tierra natal.

Para excitar al poeta, Domeyko propone "elevar la inteligencia del hombre a la idea de lo infinito". Le interesa la unión del poeta y del astrónomo, alguien que admire los "espacios estrellados", para percibir cómo lo inmóvil cambia de lugar y cómo la ciencia mide las distancias, en leguas o radios de la órbita terrestre. En lo sideral, la imaginación se pierde, dice Bello y reitera Domeyko.

Mientras argumenta, el intertexto con el cual está dialogando y de alguna manera confirmando, es Cosmos, de Humboldt, la obra más influyente en la reflexión de I)omeyko. Esta afición no puede sino deberse a la influencia de Humboldt en Bello, cuando éste conoció al enciclopedista, en Caracas, a comienzos del siglo XIX.

\section{OBSERVACIÓN E IMAGINACIÓN}

Domeyko arma en un párrafo, un paisaje, una estructura estética, una imagen visual, un icono, que convierte al hombre de ciencia en poeta. $Y$ el espacio es para él un libro que se abre: "estamos leyendo una historia de más de mil anoos de fecha", dice Bello, en su Cosmografía, citado por Domeyko. Bello sabía que mirar el cielo era mirar al pasado. Pero lo veía en términos temporales menores. Fs la vieja imagen clásica del libro de la naturaleza. 
En su concepción del espacio sideral, en las nébulas, ve Domeyko "materias que pueden haber sobrado al creador de la hechura de tantos mundos". Al mirar al cielo entonces se sorprende, en la obra, la presencia de un creador. Y eso se logra gracias al telescopio, que avizora "enjambres de mundos ya formados".

Sigue a la Cosmografia de Bello al insistir en que "veinte y mil y más estrellas cabían en un espacio que alcanzaba a un décimo del disco de la luna". Y continúa, "allí no estaba todavía el término del universo ni la ribera de los espacios". Es el momento en que poeta vería, según Bello, "una interminable cadena de sistema sobre sistema, de firmamento sobre firmamento".

La deducción es clara: "a esas distancias, apenas accesibles a la imaginación, reina y gobierna la misma ley, la misma voluntad que en la tierra". Esta es la fe del positivista, la instauración de la ley, como eje de referencias y como forma valida de aprehender la realidad.

\section{DEL LIBRO DEL CIELO AL LIBRO IDE LA TIERRA}

Al volver a la tierra, el fuego y el hielo se encuentran en la alta montaña de Chile, dice Domeyko. El geólogo y el botánico abrirán camino al poeta. Esa misma montaña tenía huellas de moluscos, de "clías anteriores a la creación del hombre y al orden actual de la naturaleza". Domeyko sale aquí del tiempo histórico y establece un tiempo mítico en el pasado.

Domeyko se muestra a la vez como un intelectual tradicional (en cuanto a la teoría del origen del universo) y como un científico moderno (en el estudio de nuestro planeta), y su discurso intenta conjugar dos órdenes. Por ello, en la roca ígnea de lo profundo de la tierra Domeyko leerá "en un gran libro la historia de lo que ha existido antes de la creación del hombre". Nuevamente toma validez la metáfora del libro de la naturaleza.

Curiosamente, Domeyko también menciona al megaterio y al mastodonte como "habitantes de Chile antes de la creación del hombre". Su idea del surgimiento del ser humano está fijada ciertamente en un momento más bien tardío.

Según Domeyko, la ciencia moderna, que ensancha los tiempos y los espacios, influye en los sentimientos y en la imaginación. Domeyko no define claramente que es el sentimiento o la imaginación. Para él, "el universo, a más de ser el conjunto de los objetos visibles que 
impresionan nuestros sentidos, es a un tiempo un sistema admirable de fuerzas que obran en la materia".

Hay aquí, primero, hay una constante identificación de lo físico con lo visible; y segundo, las fuerzas no están en la materia sino que fuera de ella. Impresiona la iclea orgánica, estructural, presente siempre en su uso de la noción de "sistema".

\section{LA INTEGRACIÓN I)EL LE GUAJE IMAGINATIVO A LA CIENCIA}

Según la perspectiva cientificista establecicla por Domeyko, la poesía ganaría si el hombre de letras conociera las fuerzas ocultas en lo cotidiano. No se olvide que él mismo quiere ser poeta y científico. Para ello, incluye en su Discurso, otra escena estética de muestra y se refiere a una gota de rocío, que puede ser descompuesta por los científicos.

Claramente Domeyko hace de la ciencia el discurso que antecede al arte. El pensamiento científico es el intertexto de la imaginación poética y del sentimiento artístico, lo cual es una reflexión positiva de un científico moderno. De acuerdo a esto, la investigación científica precede a la creación poética, y la ciencia experimental antecede a la ciencia humana.

Domeyko argumenta a favor del saber científico solbre el saber poético. El mecanismo del proceso histórico es claro: la ciencia hegemoniza a la poesía, porque la ciencia aumenta los objetos de contemplación y contribuye al ensanche del mundo. Los que trabajan el dominio del sentimiento y la imaginación no son sólo los poetas o los artistas sino que también se incluye a los filósofos, los moralistas y los oradores.

Las clefiniciones más científicas son provistas por Iomeyko, pero no las del terreno de las humanidades y así, su argumentación sigue siendo siempre la del científico. Por ejemplo, Domeyko define el mundo físico en tres instancias: como objetos de contemplación (no de transformación), como sistema de fuerzas (el carácter orgánico funcional ya notado), y como un orden con principios inmutables (con lo cual se expresa como un materialista de índole espiritualista).

\section{ENTRE EL I)ISCURSO CIENTÍFICO Y EL I)ISCURSO IMAGINATIVO}

Este esquema, basado en las funciones mentales (idealista) habla de la exactitud del raciocinio como superior a la imaginación. Otra precarie- 
dad queda a la vista cuando el mundo físico es caracterizado sólo como el mundo visible (para los creadores). Según Domeyko, en general, los artistas prefieren la brillantez del lenguaje o toman aspectos superados por la ciencia moderna.

Por eso, para él, la verdad moral o la verdad estética, están afectadas por una contradicción, del fondo, con la forma (por la inexactitud y las comparaciones sin verdad). Domeyko está en contra de una ciencia distorsionada hacia la moral o la justicia.

Otra prueba más del aporte de la ciencia, dice Domeyko, es como los idiomas modernos se enriquecen con la incorporación de nuevos términos científicos, favoreciendo de esta manera a la lingüística. Gradualmente, se incluyen más expresiones científicas en los idiomas de mayor cultura científica.

Como es obvio, la teoría de Domeyko es monofactorial, en cuanto el discurso científico hegemoniza a los otros discursos. Pero además hay una segunda propuesta, la idea de una integración, que es parte constitutiva de la argumentación presentada, y cuyo empalme con la línea central de reflexión debe ser verificado.

\section{LA ABSORCIÓN DE LO POÉTICO POR LO CIENTÍFICO}

A esta altura de la reflexión, Domeyko necesariamente comienza a referirse a los aspectos positivos de la imaginación y del discurso de las bellas artes.

La literatura sale ganando al enlazarse con la ciencia. Pero además, el hombre de ciencia debe cultivar la imaginación mediante el conocimiento de las obras clásicas. Más aún, Domeyko cree en la existencia de una inspiración o visión intelectual que se manifiesta antes del calculo y la observación, porque para él, en el descubrimiento participa la imaginación y el sentimiento.

Así pues, en el procedimiento científico es absorbido lo poético. Antes de la ciencia conclusiva hay un momento de presentimiento poético. Aquí nuevamente se plantea una visión de lo real en términos de sucesión, donde unos procesos anteceden o provocan otros. Es la visión causalista del positivismo del siglo XIX.

Cita también Domeyko a Pascal, al recordar que es necesario ver el conjunto del todo porque "todo nuestro raciocinio se reduce a ceder al sentimiento". De acuerdo a esto, antes de palpar el descubrimiento éste está revelado en la inteligencia como inspiración. Este planteamiento 
podría denominarse idealismo materialista, descle el punto de vista de la teoría del conocimiento.

Según IDomeyko, la ciencia, la filosofía, más la literatura y las bellas artes se aúnan en su movimiento hacia la verdad, lo bello y lo subli me. La literatura, especialmente, contribuye a la visión de conjunto, y no se queda en el detalle. Por otro lado, también se puede divulgar la ciencia usando un lenguaje más digno en su divulgación. Esta es otra forma de la unión de la ciencia con la literatura (donde sin embargo el lenguaje aparece subordınado e instrumentalizado).

\section{LA HUELLA IDEL CREAIDOR EN LA MATERIA CÓSMICA}

Hasta aquí Domeyko aparece compartiendo una lógica del descubrimiento en todo orden y un paradigma materialista, lo que conforma una ideología cientificista Destaca, por otro laclo, que para él. Buffon, Humboldt, Arago, Cuvier, todos científicos de nota, reúnen, al "mérito literario cierta precisión científica". Lo científico para Domeyko siempre tiene que ver con la observación visual de lo físico, la medición cuantitativa de los fenómenos, la precisión en la escala temporal.

El primer tomo de Cosmos, de Humboldt, en 1848 , se manifestó como una gran historia de las ciencias. Según Domeyko, Andrés Bello habría dicho que allí no estaba el creador, en el sistema y orden patentizados en la contemplación de las obras de I)ios.

En su Discurso Inaugural, Domeyko insiste en la idea de la reunión de la física, la poesía y la filosofía. Advierte que si la ciencia es puramente utilitaria, la poesía se mantendría a distancia. Tampoco es positivo que las artes se preocupen sólo de la forma y de los adornos sin buscar la integración con otros tipos de visión.

Segun I omeyko, así como la ciencia arriba a leyes, el arte llega a lo sublime y ambas lógicas delsen reunirse. Agrega que el sentimiento cristiano habría hecho más grande a Goethe, acentuando la importancia del poeta creyente. La posición doctrinaria de Domeyko aquí es religiosa, correspondiendo a la cle un creyente.

\section{EL TERCER I ISCL'RSO: LO REI.IGIOSO}

En el tomo II de Cosmos, cuando Humboldt toca el tema de la ciencia y la poesía, introduce el sentimiento religioso (lo que no había hecho 
en el primer volumen). Domeyko tiene una valoración positiva del cristianismo, porque desarrolló la libertad civil de los pueblos.

Humboldt realizó una glorificación de la humanidad y sus obras, al incluir en Cosmos el Salmo 103, que es un poema lírico sobre la creación de la tierra, el mar y el cielo. Algo similar acontece en el Capítulo XXXVII de Joh, donde se describen, según I omeyko, fenómenos metereológicos y temas científicos modernos.

Cuando la ciencia, la literatura y las bellas artes se elevan a la perfección tocan una misma idea, concluye Domeyko: el "conocimiento del creador, apoyado en la fe y en la revelación divina". A esta altura de su reflexión, Domeyko ha pasado de lo científico y lo poético a lo religioso.

Cabe recorclar que no es que lo poético se complemente realmente con lo científico, como aproximación de dos tipos de verdades o de discursos, sino que más bien lo poético o lo lingüístico es instrumentalizado por lo científico. Y así como la ciencia subordina a la poesía, así también posteriormente la fe subordina a la ciencia. De esta manera, se llega así a la causa primera discursiva, el creador.

\section{DE IAA MATRIZ IBINARIA A IA DIALECTICA TRINARIA}

En el marco histórico-cultural de su época, Domeyko, al contraponer dos conjuntos epistemológicos diferenciados, permite la entrada de la contradicción en su misma escritura. Lo científico y lo poético, sin embargo, no son en su reflexión dos paradigmas que se integren, sino que uno captura al otro.

Hay una estructura hegeliana en todo este planteamiento de Domeyko, si uno establece primero como tesis a la ciencia, como antítesis a la imaginación y como síntesis a la integración de ambos órdenes, en una primera formulación.

Esta estructura dialéctica se complejiza más aún si se establece que la tesis es la siguiente: que la ciencia precede a otras formas de acción y pensamiento; la antítesis, que el arte ayuda a la ciencia; y la síntesis, la actitud doctrinaria religiosa.

Con todo lo dicho en este examen del Discurso de Incorporación de Ignacio Domeyko a la Facultad de Filosofía y Humanidades de la Universidad de Chile se muestra que hay en juego en su escritura una compleja concepción epistemológica. 
En esta matriz conceptual, de carácter estructural, donde los componentes principales son los saberes, se engarzan jerárquicamente visiones físicas y metafísicas de quien fuera uno de los pilares cle la innovación y de la modernización cle la educación chilena en el siglo XIX: Ignacio Domeyko. 